\title{
Selective cement augmentation of cranial and caudal pedicle screws provides comparable stability to augmentation on all segments in the osteoporotic spine: a finite element analysis
}

\author{
Hui-Zhi Guo ${ }^{1,2 \# \wedge}$, Dan-Qing Guo ${ }^{1 *}$, Yong-Chao Tang ${ }^{2}$, De Liang ${ }^{2}$, Shun-Cong Zhang ${ }^{1,2}$ \\ ${ }^{1}$ The First Institute of Clinical Medicine, Guangzhou University of Chinese Medicine, Guangzhou, China; ${ }^{2}$ Spine Surgery Department, The First \\ Affiliated Hospital of Guangzhou University of Chinese Medicine, Guangzhou, China \\ Contributions: (I) Conception and design: HZ Guo, YC Tang, DQ Guo; (II) Administrative support: D Liang; (III) Provision of study materials \\ or patients: SC Zhang; (IV) Collection and assembly of data: HZ Guo, DQ Guo; (V) Data analysis and interpretation: HZ Guo, D Liang; (VI) \\ Manuscript writing: All authors; (VII) Final approval of manuscript: All authors. \\ "These authors contributed equally to this work. \\ Correspondence to: Shun-Cong Zhang. The First Affiliated Hospital of Guangzhou University of Chinese Medicine, 12 Airport Road, Baiyun District, \\ Guangzhou 510405, China. Email: 18122436960@163.com.
}

\begin{abstract}
Background: Cement-augmented pedicle screw instrumentation (CAPSI) has been proven to significantly increase the biomechanical stability in the osteoporotic lumbar spine. However, besides the merits, it is responsible for the inevitable cement leakage growing with more instrumented segments and volumes involved. This study aimed to compare the biomechanical performance of pedicle screws augmented on all segments with those augmented only on the cranial and caudal vertebrae selectively.

Methods: The finite element model of L3-S1 was modeled with the CT data of a healthy volunteer, the solid/ fenestrated pedicle screws from micro-CT scans of physical screws, and bone cement from the CT scans of a postoperative patient with CAPSI. Three different augmented strategies for pedicle screws were taken into consideration: augmentation at each pedicle trajectory (Model A), selective augmentation at the cranial and caudal pedicle trajectories (Model B), and pedicle trajectories without augmentation (Model C). A total of six surgical models were constructed: Models A, B, and C were subdivided into double segmental fusion from L4 to S1 (Models A1, B1, and C1) and multi-segment fusion from L3 to S1 (Models A2, B2, and C2). The Range of motion (ROM), stress on the cage, and stress on the fixed segments were compared among the six models.

Results: The ROM at the fusion segments decreased in all instrumentation models. The ROMs of Model $\mathrm{B}$ and Model A are similar in each direction, while that of Model C is significantly larger. The differences in the ROMs between Model A and Model B were noted to be less than 0.1 ${ }^{\circ}$. Compared with Models A1 and A2, the peak Von Mise stress on the cage-endplate interface and pedicle screws were slightly higher in Models B1 and B2. In contrast, the stress of Models C1 and C2 increased significantly. The compressive stress was concentrated in the screw head, the cranial and caudal screws, and rods.

Conclusions: The selective augmentation of pedicle screws is capable of providing reliable stability in short-segment posterior fixation (2- or 3-level). It could be a potential optimal procedure to minimize the associated complications of CAPSI.
\end{abstract}

Keywords: Cement augmentation; pedicle screw augmentation; osteoporotic spine; finite element analysis

Submitted Mar 06, 2020. Accepted for publication Sep 14, 2020.

doi: $10.21037 / \mathrm{atm}-20-2246$

View this article at: http://dx.doi.org/10.21037/atm-20-2246

^ ORCID: 0000-0003-3952-0030.

(c) Annals of Translational Medicine. All rights reserved. 


\section{Introduction}

Posterior instrumentation with pedicle screws is a standard protocol associated with decompression and fusion for degenerative lumbosacral disease to achieve instant stability, assist interbody fusion, and maintain sagittal alignment $(1,2)$. However, the application of pedicle screws in the osteoporotic spine is challenging, with a high risk of screw loosening, back-out, and secondary pseudarthrosis (3-5). To address fixation failure, cement-augmented pedicle screw instrumentation (CAPSI) has been one of the developed techniques. CAPSI has been proven to strengthen the mechanical force on the screw-bone interface so as to reduce the implant failure rate in both in vivo and in vitro studies $(6,7)$. It showed satisfactory outcomes in the osteoporotic spine where every pedicle trajectory was augmented with calcium phosphate cement or polymethylmethacrylate (PMMA) (8,9). However, cement leakage (CL) ranging from $11.6-82.4 \%$ has become the main concern when applying CAPSI (9-11), due to severe complications, such as nerve injury, vascular damage, pulmonary embolism, cardiac embolism, and anaphylactic shock (7-9,12).

As is acknowledged, the increasing augmented trajectories and volumes of cement used per trajectory contribute to the rising frequency of CL (9). Therefore, balancing the risk of CL and purchasing power requires us to determine the optimal strategy of augmentation. Since the greater chance of loosening and pull-out of screws lies in the cranial and caudal vertebrae, particularly in S1 due to the growing stress (13-15), it was hypothesized that selective augmentation of the upper and lower end vertebrae instead of every level could bring about the maximum benefit in reducing the failure of instrumentation and the risk of cement protrusion. This study was conducted to compare the biomechanical stability of selective augmentation of only cranial and caudal pedicle trajectories to that of traditional methods by analysis of the finite models of the lumbosacral segments.

\section{Methods}

This study was reviewed and approved by the Ethics Committee of The First Affiliated Hospital of Guangzhou University of Chinese Medicine \{NO. ZYYECK[2019]094\}. All methods in this study were carried out in accordance with the provisions of the Declaration of Helsinki (as revised in 2013). The informed consent was obtained from the volunteer and this consent was written.
A total of 7 finite-element models (FEMs) of the lumbar spine were created in this study. FEMs included: (I) the intact lumbosacral spine (L3-S1), (II) Model A1: double segmental fusion (L4-S1) with augmentation at every each pedicle trajectory, (III) Model A2: multi-segment fusion (L3-S1) with augmentation at every each pedicle trajectory, (IV) Model B1: double segmental fusion (L4-S1) with selective augmentation at cranial and caudal pedicle trajectories, (V) Model B2: multi-segment fusion (L3-S1) with selective augmentation at cranial and caudal pedicle trajectories, (VI) Model C1: double segmental fusion (L4-S1) without PMMA augmentation, and (VII) Model C2: multi-segment fusion (L3-L1) without PMMA augmentation.

\section{Construction of the intact lumbosacral model}

In the present study, a healthy adult female volunteer without any history of lumbar diseases was selected and the data of her CT scans (AQUIRRON 64, Toshiba, Japan) with the thickness of $0.625 \mathrm{~mm}$ per slice was provided by the Department of Radiology at The First Affiliated Hospital of Guangzhou University of Chinese Medicine. The tomography images were stored in Digital Imaging and Communications in the Medicine (DICOM) format.

The collected raw data in DICOM format were imported into Mimics research 19.0 (Materialize, Leuven, Belgium) for three-dimensional reconstruction. Subsequently, the 3D model generated by Mimics was imported into Geomagic Studio 2013 (3D Systems Corporation, South Carolina, USA). The spikes and the features were deleted, smoothing was performed with a polygon mesh, and the triangles were made uniform in size. Then, a patch was generated by the following tools: Construct Patches, Grid and Fit Surfaces. The smoothed model was imported into SolidWorks 2017CAD (SolidWorks Corporation, Concord, MA, USA). The cancellous bone, cortical bone, annulus fibrosus, nucleus pulposus, endplate, and articular cartilage parts were created in the Parts Interface window. The nucleus pulposus constituted approximately $44 \%$ of the disc volume (16), the thickness of the cortical bone was approximately $0.5 \mathrm{~mm}$ (16), and the cartilaginous endplates were modeled to be approximately $1 \mathrm{~mm}$ thick (17). The initial gap between the articulating surfaces was based on computed tomography images and was approximately 0.3 $0.6 \mathrm{~mm}$. The parts were integrated into an intact lumbar model. Finally, the intact lumbosacral model was imported into ANSYS Workbench 17.0 (ANSYS, Ltd., Canonsburg, 

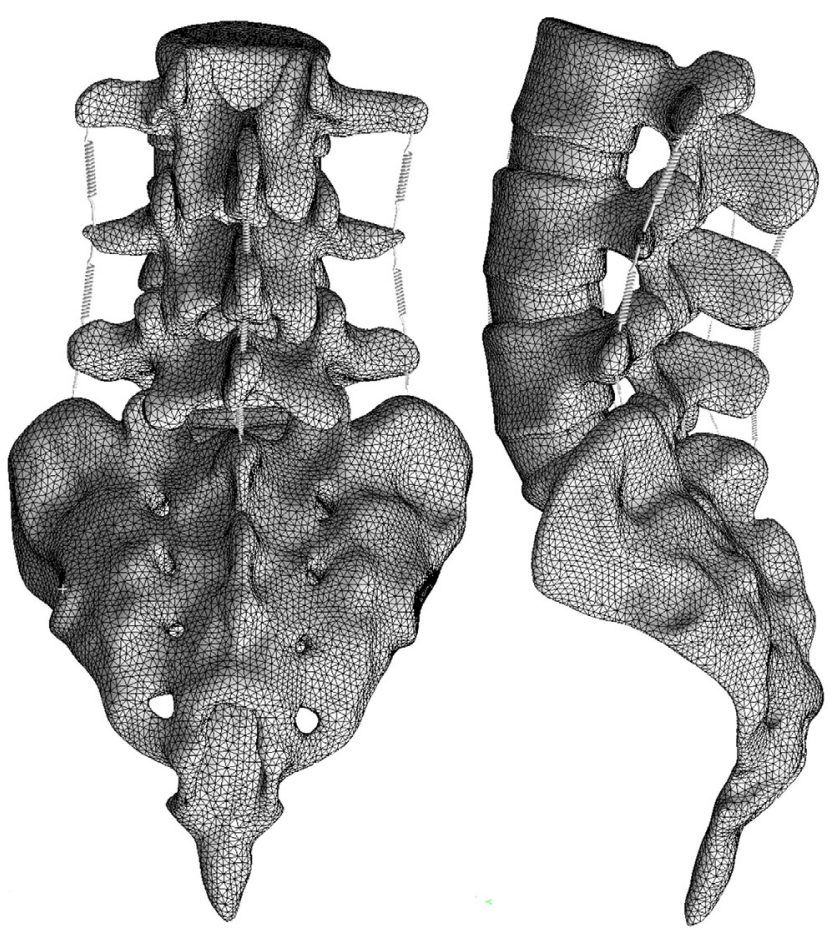

Figure 1 Intact lumbar finite-element model with no instrumentation.

Pennsylvania, USA) for setting material properties, defining major ligaments, and generating mesh (Figure 1).

\section{The model of the pedicle screw and bone cement}

To simulate the characteristics of the material object accurately, the model of the solid pedicle screw and fenestrated pedicle screw were constructed based on Micro-CT scanner images (Skyscan 1172, Bruker microCT, Belgium) with a slice thickness of $15 \mathrm{um}$, Similarly, the realistic three-dimensional model of screws were reconstructed by using the Mimics research 19.0, Geomagic Studio 2013, and SolidWorks 2017CAD. Then, a postoperative data of CT scans from a patient who received posterior instrumentation with PMMA-augmented fenestrated pedicle screws was randomly selected for constructing the cement model with the same software. The length and outer diameter of the pedicle screws (DePuy Synthes, California, USA) were 50 and $6.0 \mathrm{~mm}$, respectively. The fenestrated screws were used in augmented vertebrae and the solid screws were used in non-augmented vertebrae. The volume of bone cement was approximately $1.73 \mathrm{~cm}^{3}$ per trajectory and distributed in a lump pattern. The model of screws and bone cement were shown in Figure 2.

\section{Construction of instrument models in different augmentation fashions}

Based on the physical rod, crosslink, and cage, the model of rod, crosslink, and cage were constructed in the Parts Interface window of SolidWorks 2017CAD. The outer diameter of the rod was $5.5 \mathrm{~mm}$. The length and height of the cage were 24 and $12 \mathrm{~mm}$. Subsequently, unilateral transforaminal lumbar interbody fusion (TLIF) was performed in right to remove the facet joint, articular cartilage, annulus fibrosus, endplate, and nucleus pulposus. The cage, screws, cement, rod, and crosslink were assembled into the lumbosacral spine model to construct six surgical models (Figure 3). The cage was designed to locate in the right close to the para-central line of the intervertebral space based on the images of CT scans. To decrease variation caused by the locations of cage, screw, bone cement, and crosslink, all these parts were set in the same location among different models.

\section{Material properties, boundary and loading conditions}

The mesh model generated in SolidWorks 2017CAD was imported into ANSYS Workbench 17.0 for finite element analysis. Previous studies were referenced to set the material properties of osteoporotic cortical bone, osteoporotic cancellous bone, articular cartilage, endplates, annulus fibrosus, nucleus pulposus, cages, bone cement, and internal fixation $(18,19)$ (Table 1). The ligaments were simulated using spring elements that only stressed by pulling force. Spring stiffness were assumed from a previous validation experiment [spring stiffness $=$ (modulus of elasticity $\times$ crosssection)/average length] (20). The contact type between the models was defined in the connection, where the contact type of facet joint was "frictional" and the frictional coefficient was 0.1 (19); the remaining contact types were set to the "bonded" mode. To enhance the accuracy of calculation, the type and size of mesh in the models are controlled: the mesh type is set as tetrahedron mesh, the size of articular cartilage mesh is $0.5 \mathrm{~mm}$, and the remaining objects are $2.0 \mathrm{~mm}$. Finally, the boundary and loading conditions of the seven models were set: with all degrees of freedom of the sacrum was constrained throughout the whole analysis, a $150-\mathrm{N}$ vertical axial preload were imposed on the superior surface of $\mathrm{L} 3$ and a pure moment of $10 \mathrm{~N} / \mathrm{m}$ were applied on the L3 superior surface along the radial 

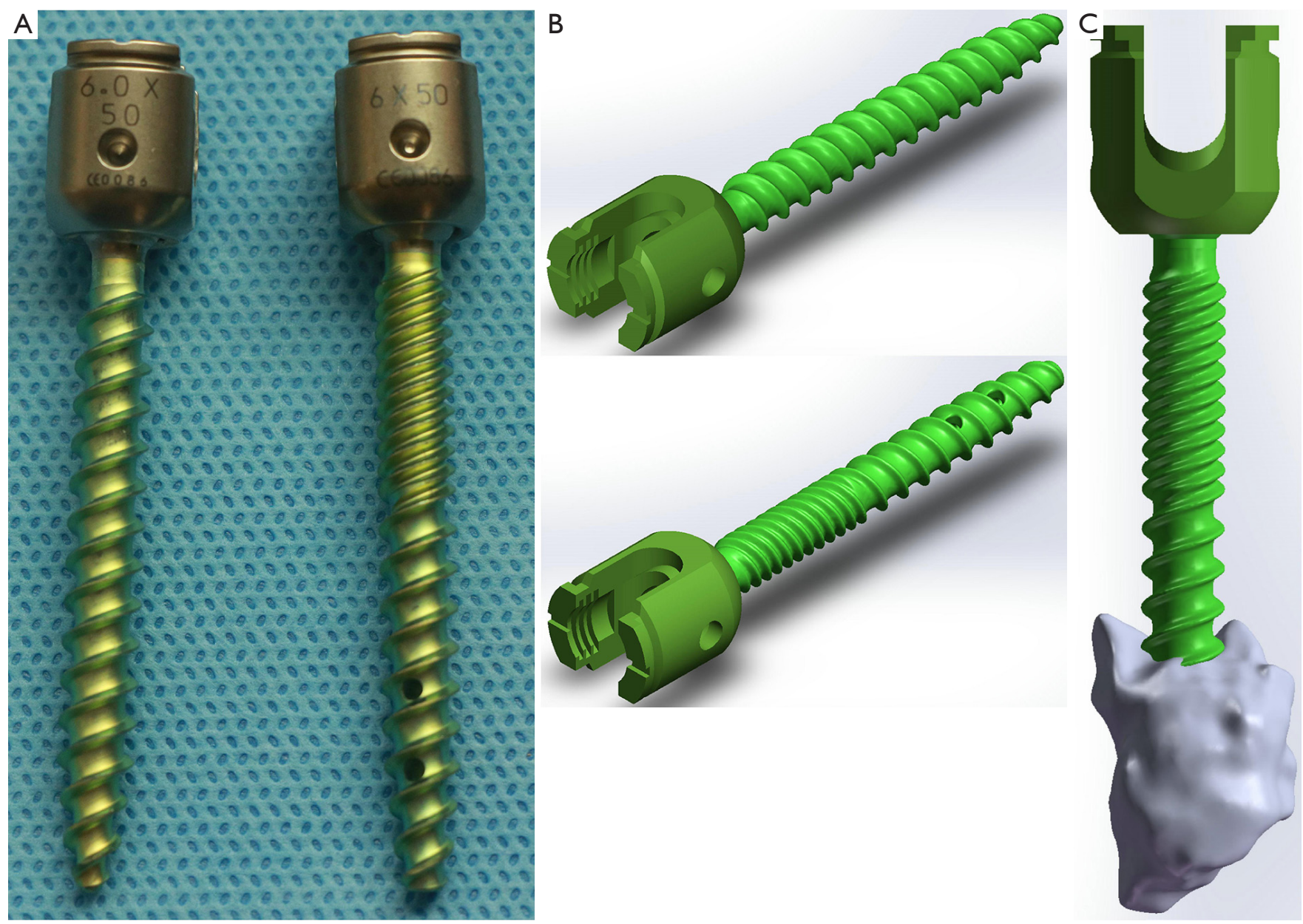

Figure 2 To simulate the characteristics of the material object accurately, the model of solid and fenestrated pedicle screws were constructed based on micro-CT scanner images (the solid screws were used in the non-augmented vertebrae and the fenestrated screws were used in the augmented vertebrae). The model of bone cement was developed from the CT scans of a postoperative patient with CAPSI. (A) Physical solid pedicle screw (left) and fenestrated pedicle screw (right), (B) a model of a solid pedicle screw (up) and a fenestrated pedicle screw (down), (C) a model of the cement-augmented fenestrated pedicle screw. CT, computed tomography; CAPSI, cement-augmented pedicle screw instrumentation.

direction to simulate 6 different physiological motions: flexion, extension, left lateral bending, right lateral bending, left rotation, and right rotation. The range of motion (ROM), the peak Von Mises stress of cage, and the stress of fixation under different loading conditions were compared to investigate the biomechanical stability of various instruments.

\section{Results}

\section{Validation of the intact lumbosacral spine model}

The finite element (FE) solution was composed using a model with 630,212 nodes and 393,835 elements. We compared our ROM data of the intact lumbosacral spine model to previous cadaveric and $\mathrm{FE}$ studies with similar loads (21-23). The results indicated that the level of spinal kinematics of the current model was in accordance with the reported data (Figure 4). The overall ROMs of flexion, extension, bending, and rotation were $21.3^{\circ}, 12.35^{\circ}, 13.99^{\circ}$, and $6.11^{\circ}$, respectively.

\section{Range of motion}

Under the combined loading of $150 \mathrm{~N}$ and $10 \mathrm{~N} / \mathrm{m}$, 


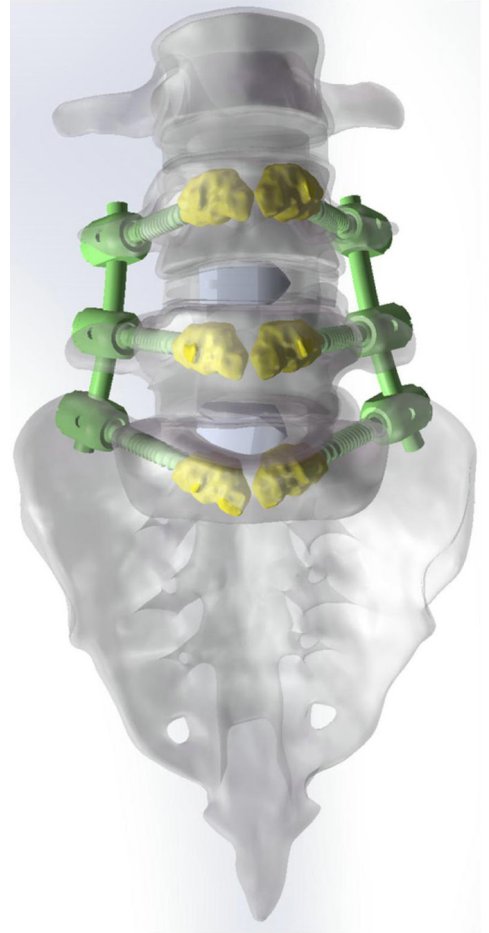

Model A1

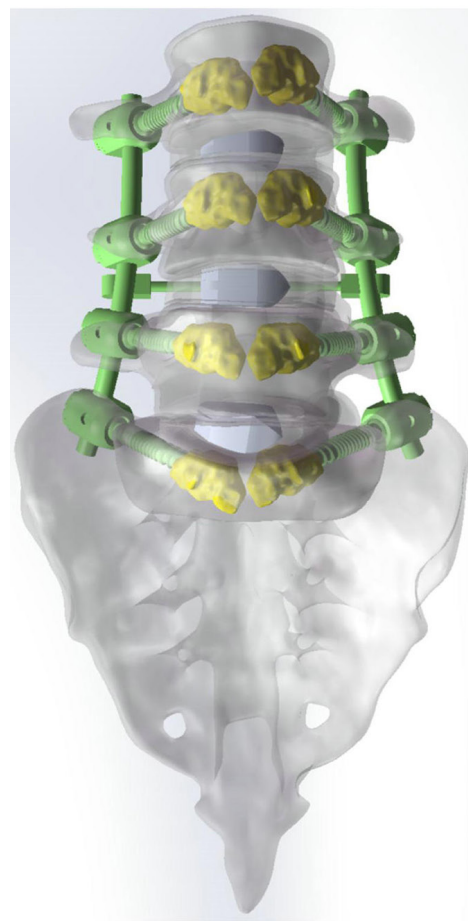

Model A2

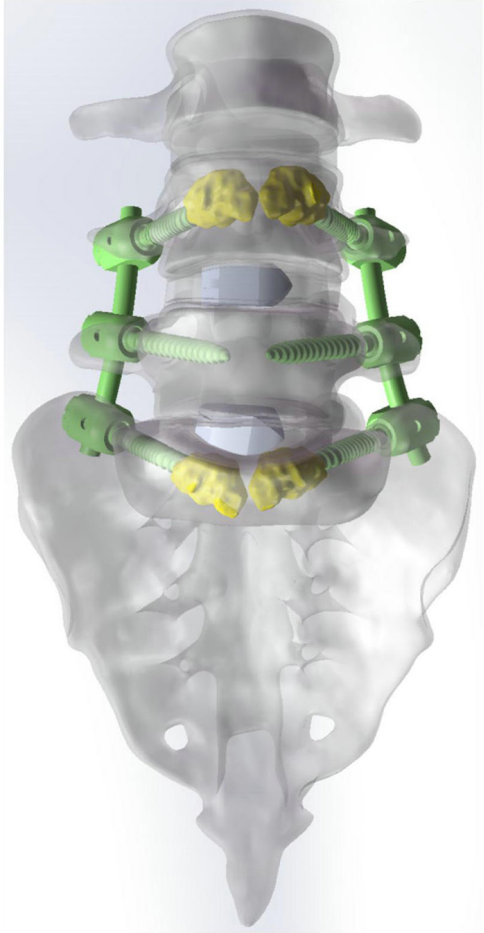

Model B1

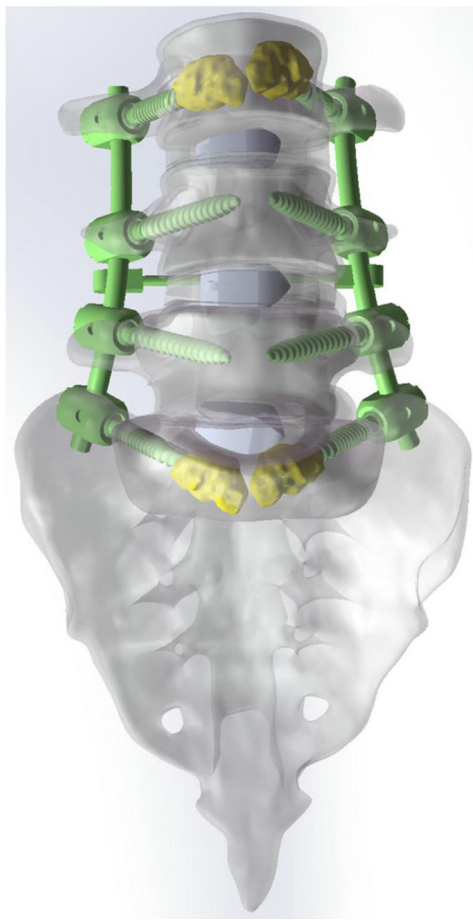

Model B2

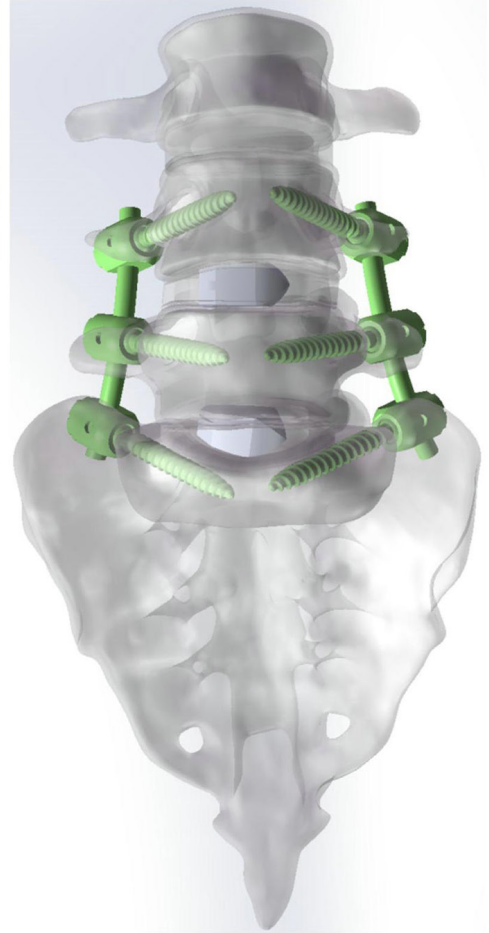

Model C1

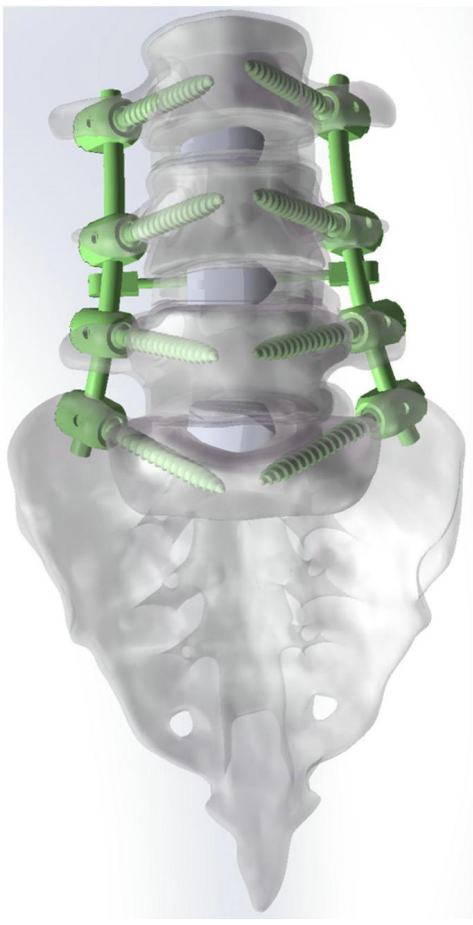

Model C2

Figure 3 Geometric instrument models following double- and multi-level transforaminal lumbar interbody fusion and pedicle screw fixation (to maintain consistency, the same location of cages, screws, rods, and bone cement were used in the different models). Model A1/ A2: PMMA on all segments; Model B1/B2: PMMA on the cranial and caudal vertebrae selectively; Model C1/C2: without augmentation. PMMA, polymethylmethacrylate. 
Table 1 Material properties used in the finite-element model

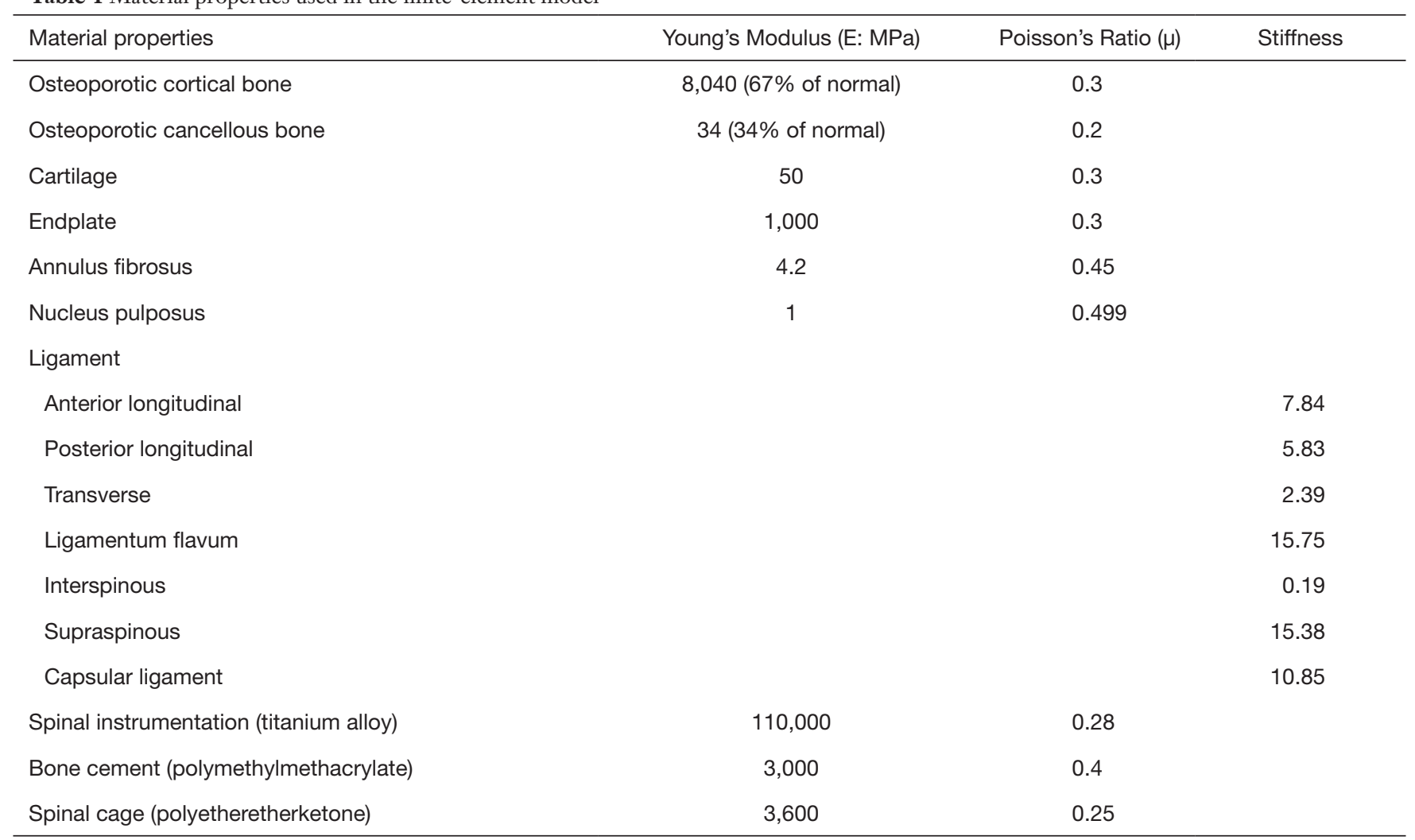

the predicted data of all ROMs of the surgical models decreased when compared to the intact model. The ROMs of Model B and Model A were similar in each direction, while that of model $\mathrm{C}$ was significantly larger. The differences in the ROMs between Model A and Model B were less than $0.1^{\circ}$ (Figure 5). When compared with the ROM in Model A1, the flexion moment in Model B1 and Model C1 increased by $4.14 \%$ and $18.62 \%$, respectively. In extension, increased by $3.28 \%$ and $9.84 \%$, respectively. In the left bending, increased by $2.4 \%$ and $8.8 \%$, respectively. In right bending, increased by $1.41 \%$ and $6.38 \%$, respectively. In left rotation, increased by $4.55 \%$ and $22.72 \%$, respectively. In right rotation, increased by $7.25 \%$ and $15.94 \%$, respectively. Compared with Model A2, the ROM in Model B2 increased by $2.68 \%, 1.71 \%, 1.97 \%, 2.55 \%, 5.10 \%$, and $9.52 \%$ during flexion, extension, left bending, right bending, left rotation, and right rotation, respectively. While the ROM in Model C2 significantly increased by $12.08 \%, 14.86 \%$, $20.39 \%, 15.29 \%, 37.76 \%$, and $23.81 \%$ during flexion, extension, left bending, right bending, left rotation, and right rotation, respectively.

\section{The stress of the Cage}

Upon comparison with Models A1 and A2, the peak von Mises stress of the cage was found to be slightly higher in Model B1 (increased by 6.92-15.99\%) and B2 (increased by $2.05-14.47 \%$ ) at the cage-endplate interface under all loading conditions (Figure 6). In contrast, the peak von Mises stress of Models C1 and C2 were observed to increase by $19.43-47.24 \%$ and $22.78-38.92 \%$ for all motions, respectively. The largest cage stress in Models A, B, and $\mathrm{C}$ was found in flexion: the maximum stress of the cage reached 55.13, 58.95, and 65.84 MPa in Models A1, B1, and $\mathrm{C} 1$; and 57.33, 65.02, and 79.68 MPa in the Models A2, B2, and $\mathrm{C} 2$, respectively.

\section{Internal fixation stress}

A similar change in the internal fixation stress was observed. Upon comparison with Model A, a significantly smaller change in the instrument stress was present in Model B. In Models B1 and B2, the maximum von Mises stress of the instrument increased by $10.61-16.50 \%$ and 

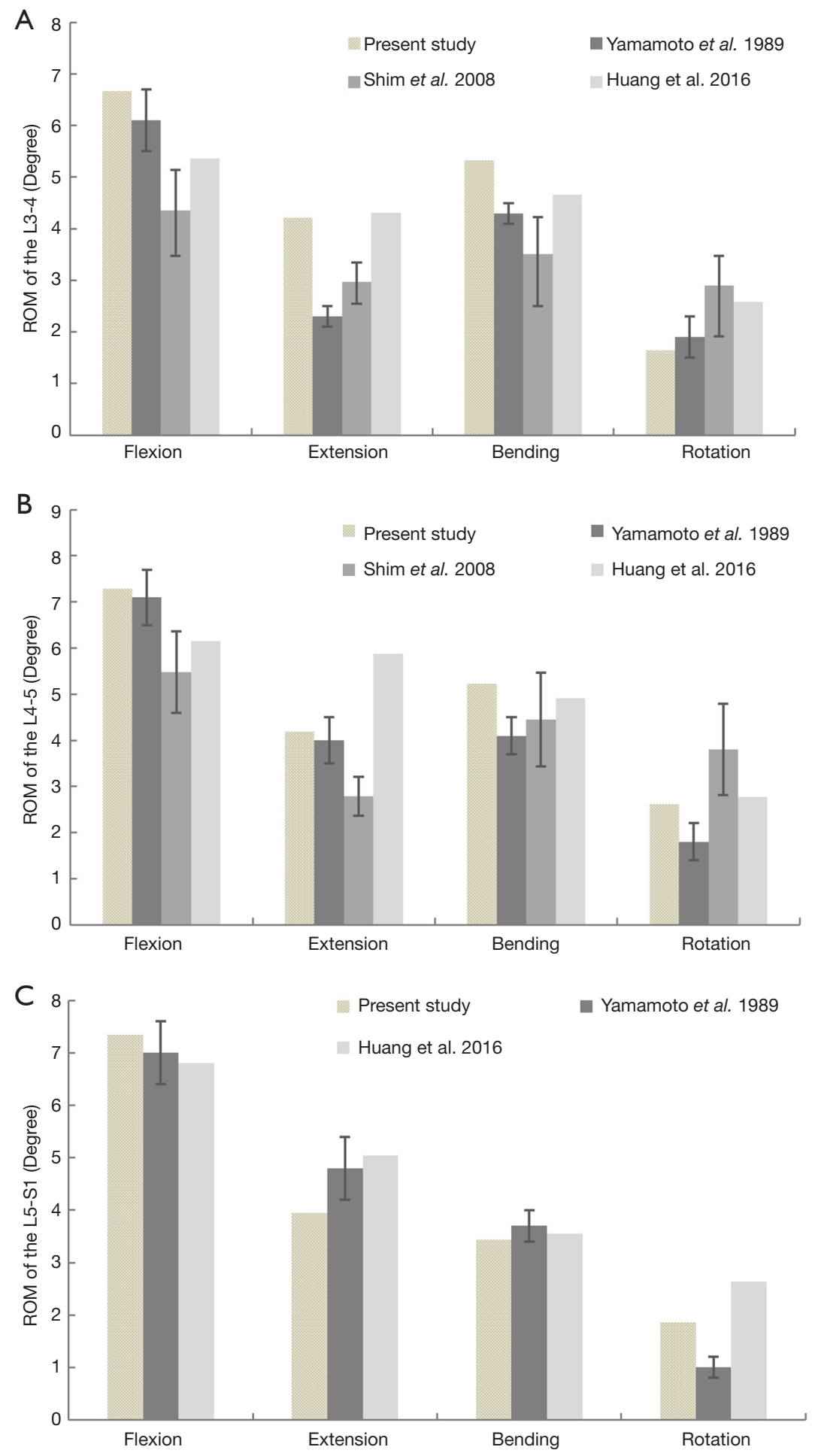

Figure 4 Comparison of the ROM between the intact model and previously published studies at L3-4 (A), L4-5 (B), and L5-S1(C) levels (ROM of L5-S1 were not provided in Shim's study). ROM, range of motion. 


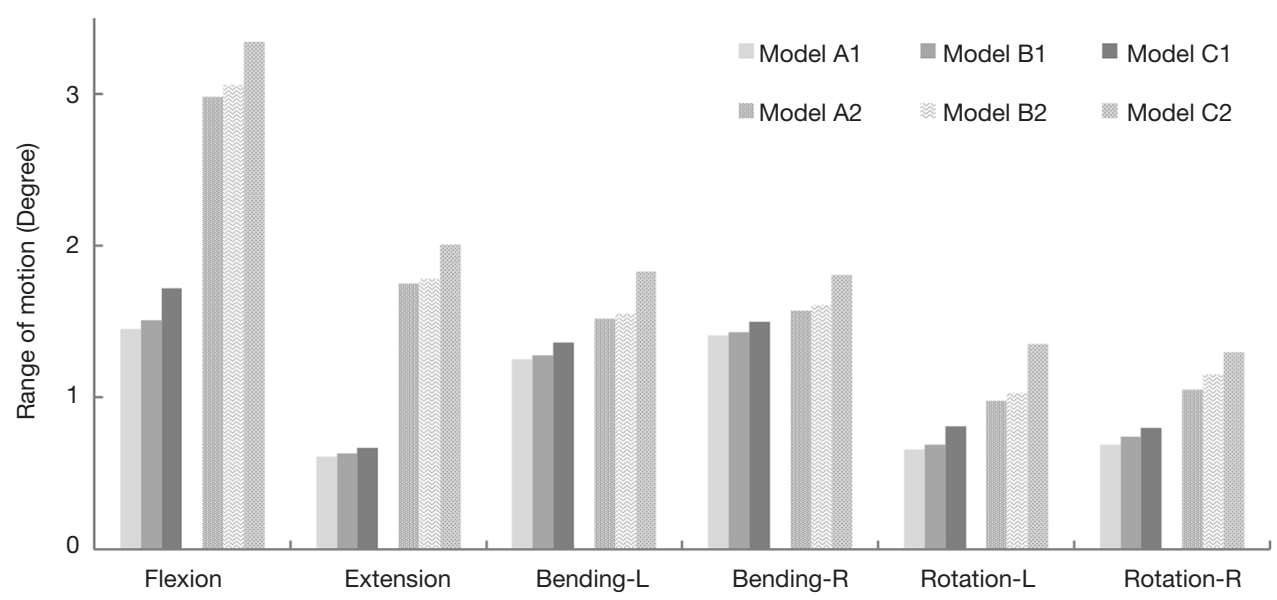

Figure 5 Comparison of the range of motion at fixation segments in Models A, B, and C for different loading motions.

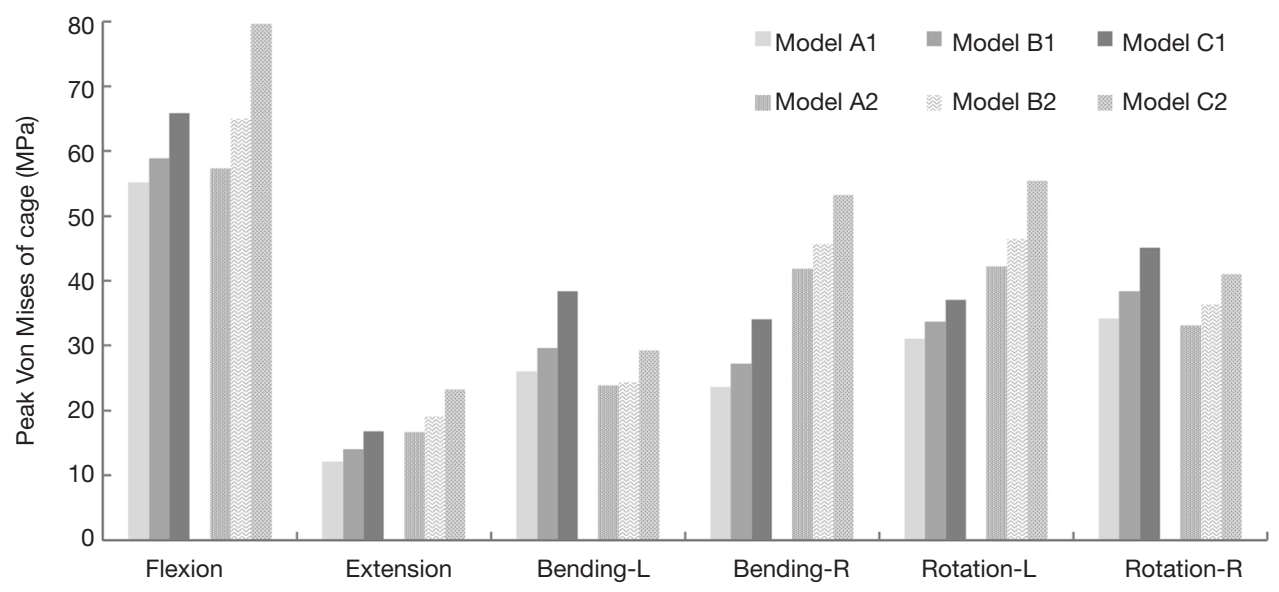

Figure 6 Stress exerted on the cage in Models A, B, and C.

9.75-21.64\% compared to Models A1 and A2, whereas the changes in Models C1 and C2 increased by 40.79$54.66 \%$ and $39.78-57.07 \%$ compared with Models A1 and A2 under different moments (Figure 7). In all the surgical models, the maximum von Mises stress of internal fixation was significantly lower than the yield strength of the titanium alloy screw/rod (897-1,034 MPa) (24). Compared with screws in the middle level, the stress on the cranial and caudal pedicle screws was larger in most loading conditions (Table 2). The stress distribution on the pedicle screw showed that the stress was concentrated in the screw head, the cranial and caudal screws, and rods (Figure 8).

\section{Discussion}

With a rapid increase in the aging population, the demand for spinal surgeries has grown markedly in elderly patients. Epidemiological studies showed that the average age of patients treated by surgery significantly increased from 54.6 years in 2004 to 63.7 years in 2015 in Asia $(25,26)$. Spinal fusion with posterior pedicle screw fixation is one of the most common strategies for lumbar degenerative diseases $(26,27)$; however, it is challenged with the increasing incidence of early implant failure or cutout (28). To strengthen the purchase, CAPSI is one of the procedures developed; it has received growing attention due to its 


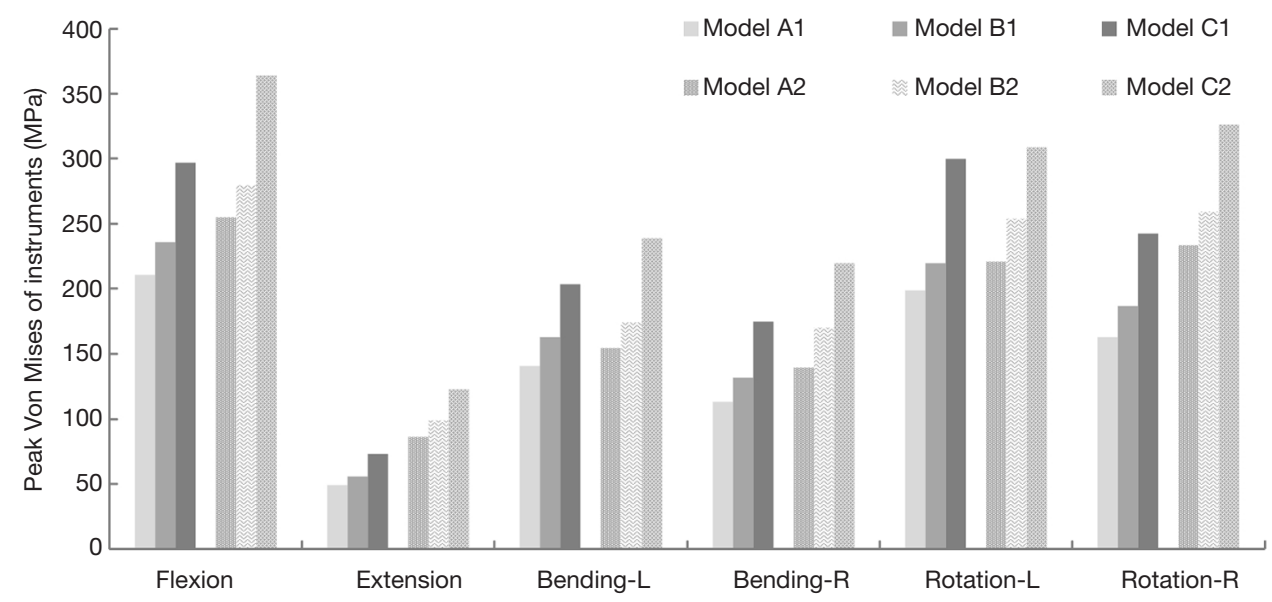

Figure 7 Screw stress at the fixation segments in Model A, B, and C.

Table 2 The comparison of pedicle screw stress in different models (MPa)

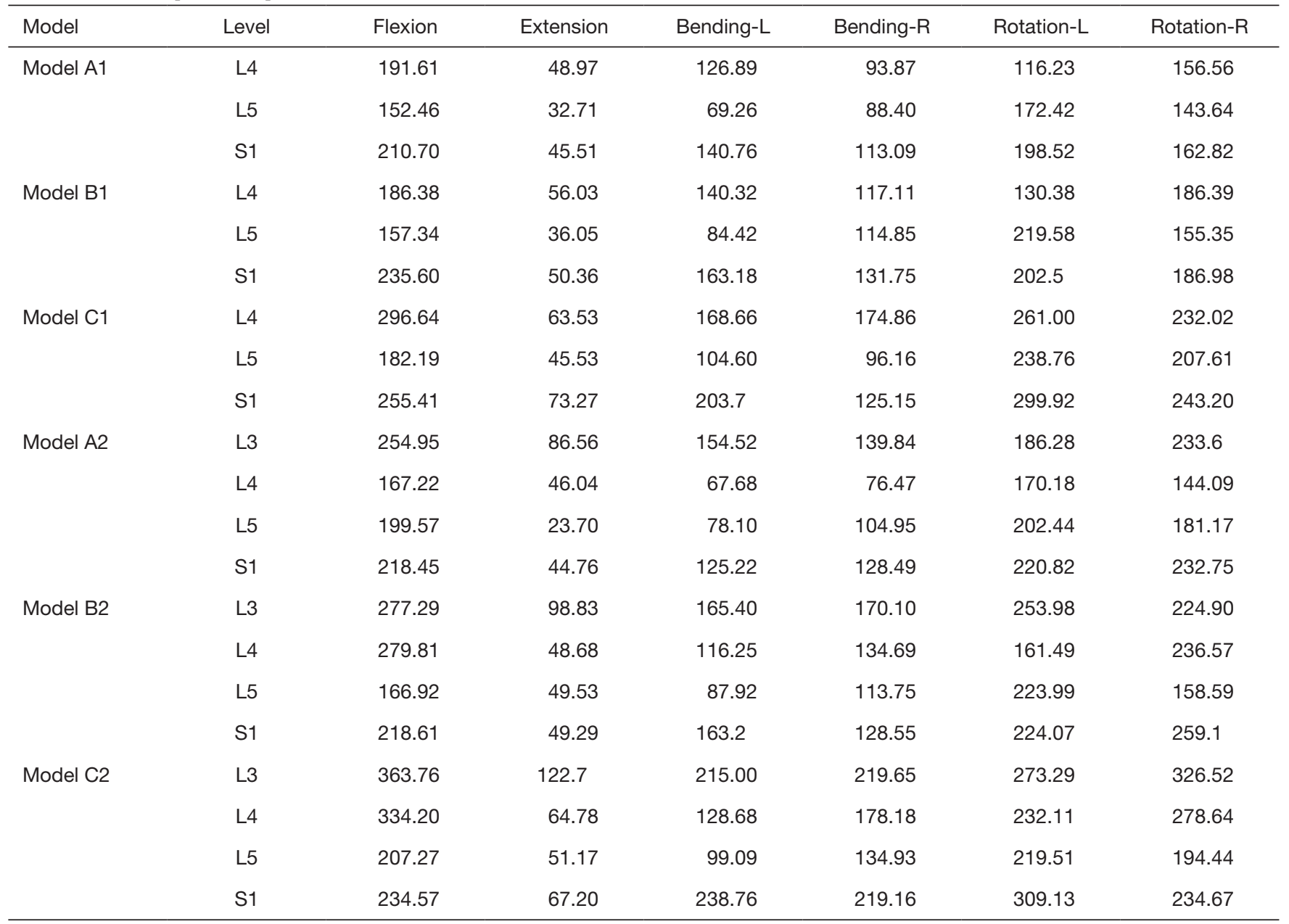




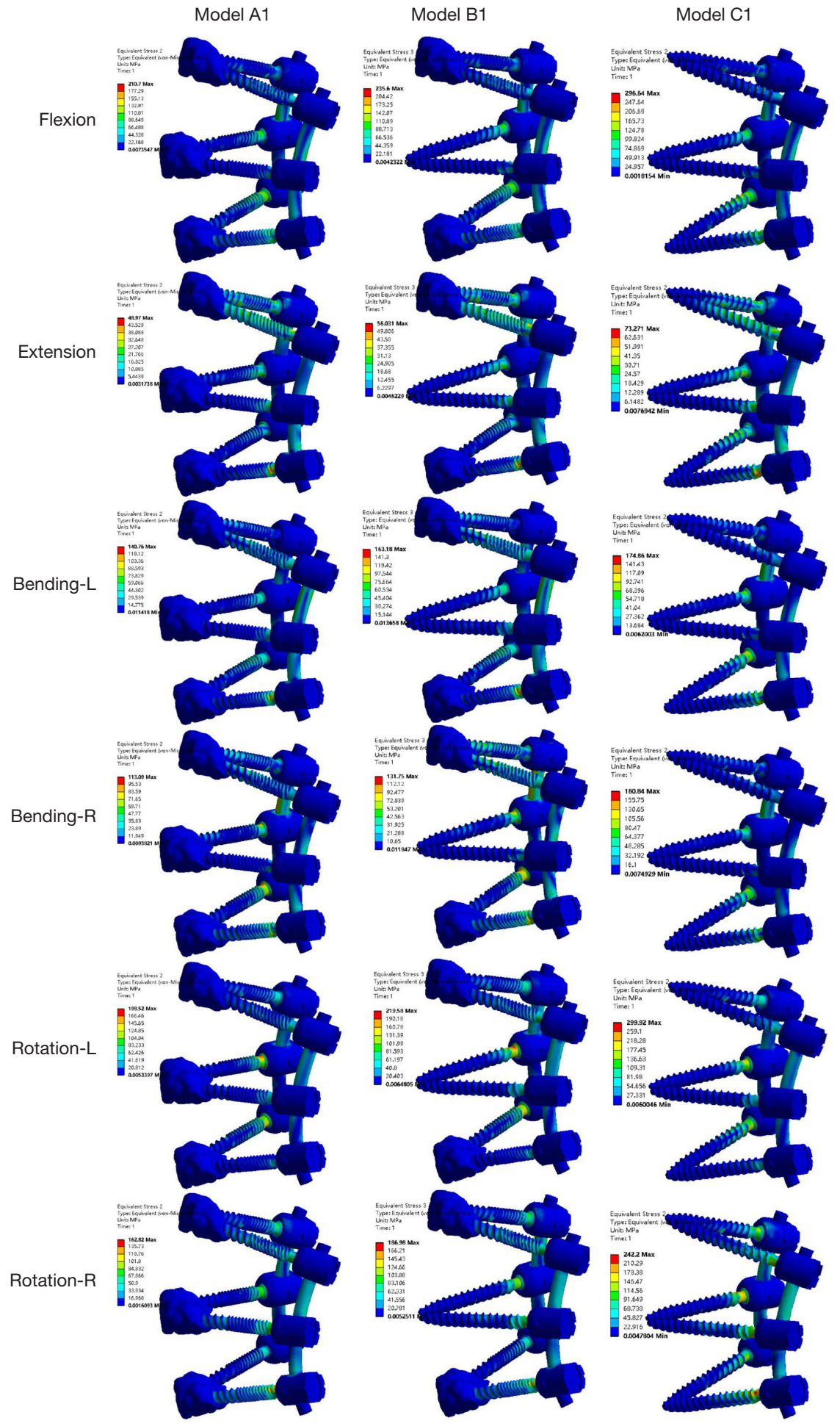




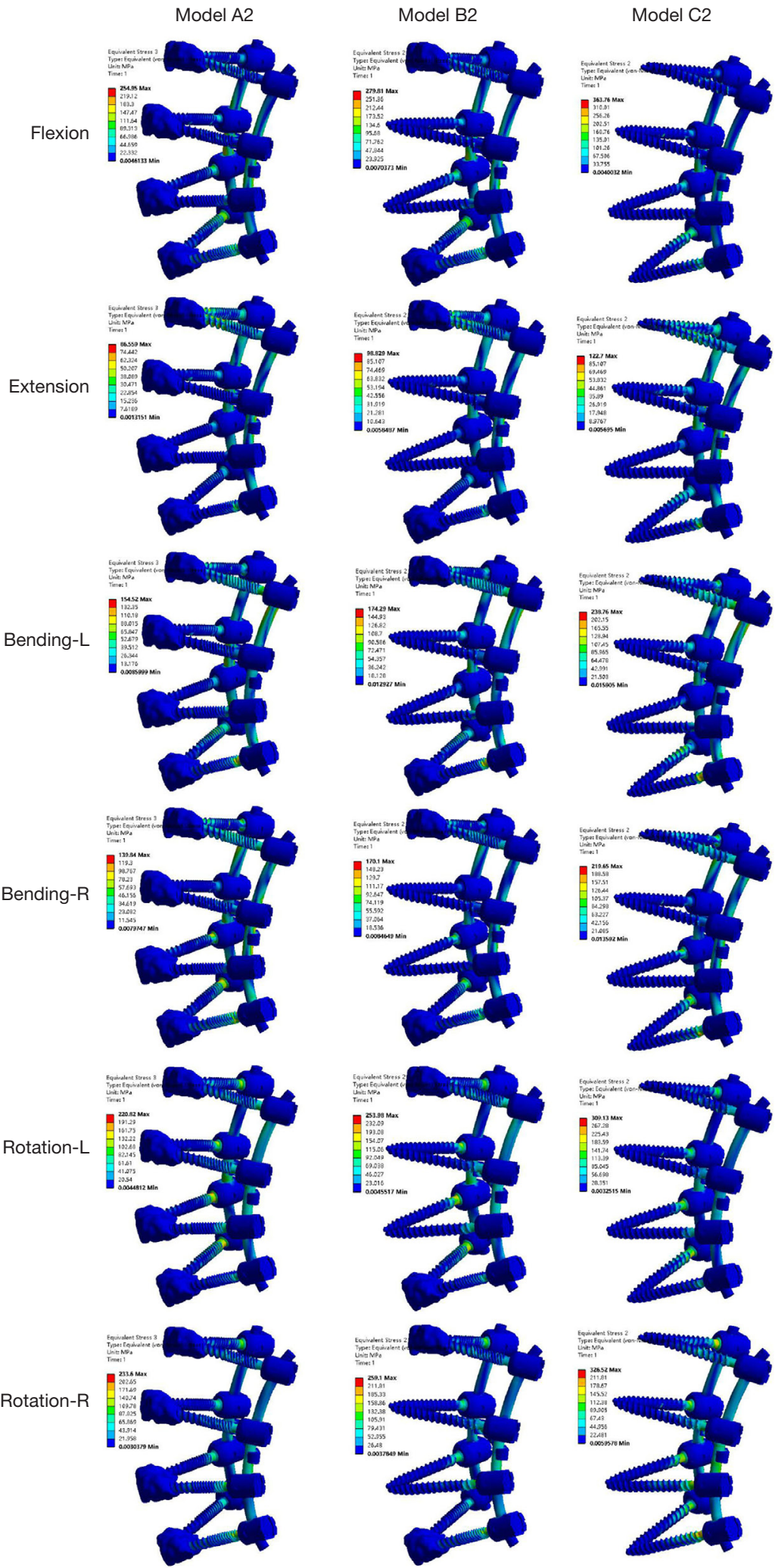

Figure 8 Stress distribution on the pedicle screw shows that the stress is mainly distributed at the screw head, the cranial and caudal screws, and rods. (A) Double segmental fusion model, (B) multi-segment fusion model. 
rigid bone fixation and handy surgical procedure (29,30). However, the major disadvantage of CAPSI is related to cement leakage, which probably leads to radicular compression symptoms, cement embolism, infection, and anaphylactic shock $(7-9,12)$. Additionally, a larger number of cement-augmented segments may also increase the cost of surgery. Therefore, these deficiencies limit the popularization of CAPSI in medical centers around the world.

To reduce the cement leakage, the protocol has been optimized by minimizing the cement volume per trajectory and the number of augmented screws $(31,32)$, using highviscosity bone cement and low application pressure $(8,33)$, by placing the tip of the fenestrated screw close to the lateral side of the vertebral body (9), and maintaining a positive end-expiratory pressure (PEEP) during cement injection (34). In addition, Erdem (31) reported a novel strategy for CAPSI recently wherein the most proximal trajectories were selectively cement-augmented, which was proven in thirty-one patients with degenerative spinal pathologies to possess compatible fixation strength as nonselectively augmenting does, moreover, with less cement leakage. However, this retrospective study has several limitations, such as the diversity of enrolled cases including various types of diseases, different lumbar instrumented segments, and multiple methods of fixation. In addition, there is a lack of a standard algorithm in the selection of the strategic vertebrae: the number of cemented pedicle screws to be placed proximally was only determined by the surgeon's experience. Therefore, in the present study, we used FEM analysis to control the consistency of the vertebral body, screws, and bone cement in different models.

Previous studies have reported that the most common site of screw loosening was identified at the cranial or caudal vertebra in lumbosacral degenerative diseases with short-segment posterior internal fixation ( $\leq 3$ levels) (15). Our previous research also showed that there was $20-24 \%$ of S1 screw loosening in the osteoporotic spine with a degenerative disease (15). Uehara et al. (13) analyzed 120 patients with adolescent idiopathic scoliosis (AIS) undergoing pedicle screw fixation. The results showed loosening rates of $9.6 \%$ at the cranial vertebra, $5.4 \%$ at the caudal vertebra, and only $2.6 \%$ at other levels. Wu (35) and Li et al. (14) also found that instrument failure (broken screw or rod) usually occurs at the lowest segment in cases of multilevel pedicle screw fixation. Therefore, selectively augmenting the upper and lower vertebrae is a potential way to optimize the strategy.

Finite element analysis is a complementary technique for cadaveric tests to characterize the complex biomechanical mechanism of the spine. In the present study, we used the finite-element method to compare the biomechanical properties of pedicle screws augmented by PMMA in every pedicle trajectory (Model A) with those that did selectively in cranial and caudal ones (Model B) or without PMMA augmentation (Model C). The results showed that although the stress of the cage and pedicle screws were found to be slightly high in Model B, differences in the ROM between Models A and B were not significant at less than $0.1^{\circ}$ under all loading conditions. In contrast, the peak von Mises stress on the cage-endplate interface and the instrument of Model C increased significantly. The result indicated that selectively cement augmenting the pedicle screws both cranially and caudally could provide comparable postoperative stability and not obviously increase the risk of cage subsidence (Figure 9). The largest stress on the pedicle screw in Model B was found when the loading flexion and rotation forces were 279.81 $\mathrm{MPa}$ and 259.1 $\mathrm{MPa}$, respectively. It was probably affected more by the complete resection of one side of the facet joints. Moreover, the stress on the pedicle screws was intensively distributed on the cranial and caudal pedicle screws, which probably resulted from their endurance of greater mechanical forces and contributed to maintaining the stability of the surgical segments without augmenting other vertebrae as well as explaining the nature of screw loosening mainly occurring at the upper and lower vertebrae.

Although this study is based on the physical models of the lumbar spine, pedicle screws, and bone cement, there are still some limitations. First, the variation in the shape, location, and dosage of bone cement may affect the purchase strength of CAPSI; additionally, only one situation was used to construct the models. Second, this study did not analyze the range of mild to severe osteoporotic models, which may lead to selective bias. Moreover, the suitability of selective augmentation for long segmental fixation remains unclear. Further investigations both in vitro and in vivo should be conducted in the future to reach a more precise conclusion.

\section{Conclusions}

Selectively cement augmenting pedicle screws both cranially and caudally is capable to provide comparable stability as non-selectively for lumbosacral degenerative diseases 

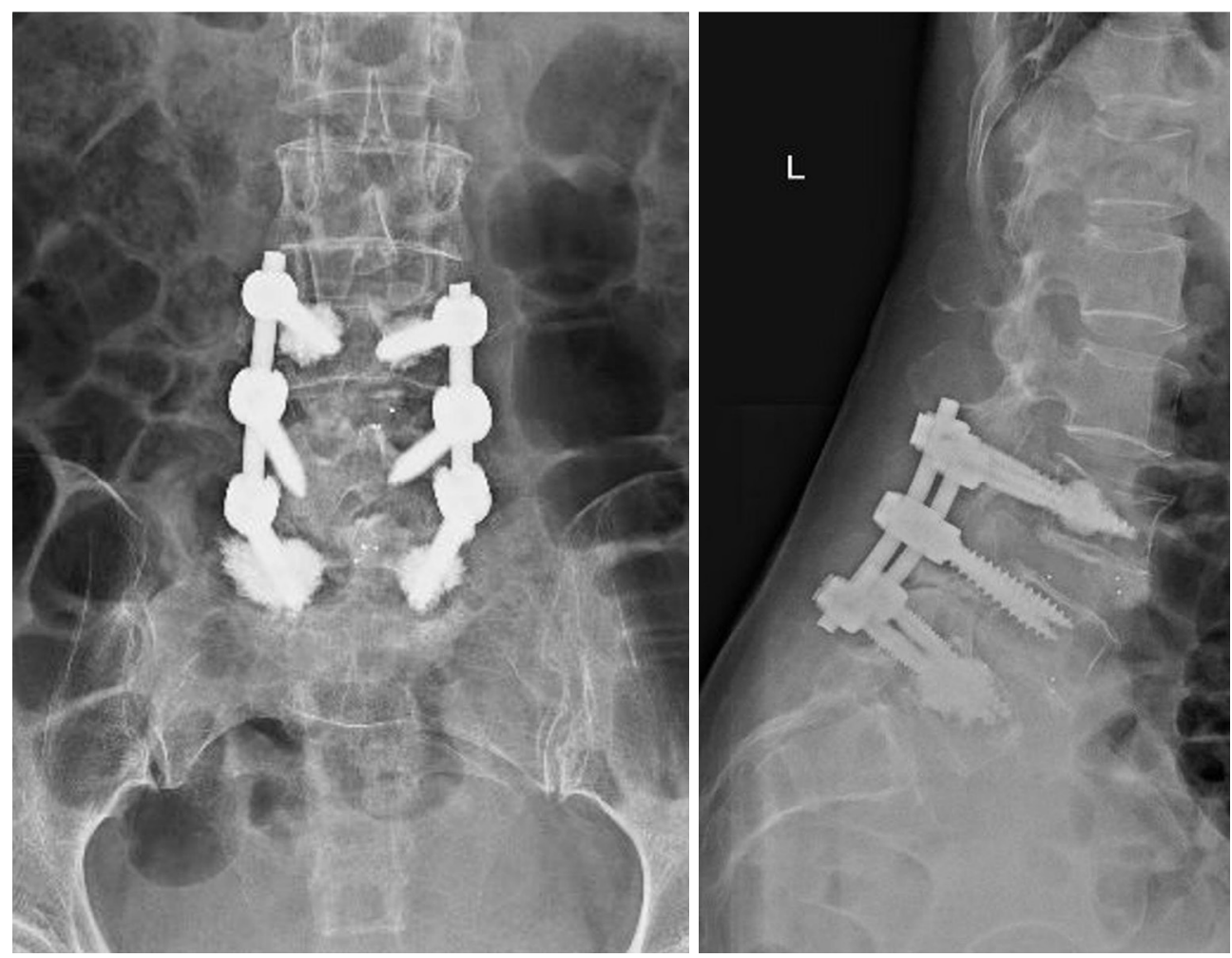

Figure 9 Selective augmentation of the upper and lower vertebrae is a potential way to balance the amount of cement and the stability of the surgical segment.

with less risk of cement leakage. Although with slightly increasing stress on cages and screws, it could be a potential optimal procedure to minimize associated complications of CAPSI.

\section{Acknowledgments}

Funding: This study is supported by the project of Traditional Chinese Medicine Bureau of Guangdong Provincial (20203004). The funders had no role in study design, data collection or analysis, decision to publish, or preparation of the manuscript.

\section{Footnote}

Data Sharing Statement: Available at http://dx.doi. org/10.21037/atm-20-2246

Peer Review File: Available at http://dx.doi.org/10.21037/ atm-20-2246
Conflicts of Interest: All authors have completed the ICMJE uniform disclosure form (available at http://dx.doi. org/10.21037/atm-20-2246). The authors have no conflicts of interest to declare.

Ethical Statement: The authors are accountable for all aspects of the work in ensuring that questions related to the accuracy or integrity of any part of the work are appropriately investigated and resolved. All methods in this study were carried out in accordance with the provisions of the Declaration of Helsinki (as revised in 2013). This study was reviewed and approved by the Ethics Committee of The First Affiliated Hospital of Guangzhou University of Chinese Medicine \{NO. ZYYECK[2019]094\}. The informed consent was obtained from the volunteer and this consent was written.

Open Access Statement: This is an Open Access article distributed in accordance with the Creative Commons Attribution-NonCommercial-NoDerivs 4.0 International 
License (CC BY-NC-ND 4.0), which permits the noncommercial replication and distribution of the article with the strict proviso that no changes or edits are made and the original work is properly cited (including links to both the formal publication through the relevant DOI and the license). See: https://creativecommons.org/licenses/by-nc-nd/4.0/.

\section{References}

1. Knez D, Mohar J, Cirman RJ, et al. Variability Analysis of Manual and Computer-Assisted Preoperative Thoracic Pedicle Screw Placement Planning. Spine (Phila Pa 1976). 2018;43:1487-95.

2. McMordie JH, Schmidt KP, Gard AP, et al. Clinical and Short-Term Radiographic Outcomes of Minimally Invasive Transforaminal Lumbar Interbody Fusion With Expandable Lordotic Devices. Neurosurgery 2020;86:E147-55.

3. Weiser L, Huber G, Sellenschloh K, et al. Insufficient stability of pedicle screws in osteoporotic vertebrae: biomechanical correlation of bone mineral density and pedicle screw fixation strength. Eur Spine J 2017;26:2891-7.

4. Liu D, Sheng J, Luo Y, et al. Biomechanical comparative study of the stability of injectable pedicle screws with different lateral holes augmented with different volumes of polymethylmethacrylate in osteoporotic lumbar vertebrae. Spine J 2018;18:1637-44.

5. Ding Q, Chen J, Fan J, et al. Effect of zoledronic acid on lumbar spinal fusion in osteoporotic patients. Eur Spine J 2017;26:2969-77.

6. Weiser L, Huber G, Sellenschloh K, et al. Time to augment?! Impact of cement augmentation on pedicle screw fixation strength depending on bone mineral density. Eur Spine J 2018;27:1964-71.

7. Janssen I, Ryang YM, Gempt J, et al. Risk of cement leakage and pulmonary embolism by bone cementaugmented pedicle screw fixation of the thoracolumbar spine. Spine J 2017;17:837-44.

8. Martín-Fernández M, López-Herradón A, Piñera AR, et al. Potential risks of using cement-augmented screws for spinal fusion in patients with low bone quality. Spine J 2017;17:1192-9.

9. Guo HZ, Tang YC, Guo DQ, et al. The cement leakage in cement-augmented pedicle screw instrumentation in degenerative lumbosacral diseases: a retrospective analysis of 202 cases and 950 augmented pedicle screws. Eur Spine J 2019;28:1661-9.
10. Moussazadeh N, Rubin DG, McLaughlin L, et al. Shortsegment percutaneous pedicle screw fixation with cement augmentation for tumor-induced spinal instability. Spine J 2015;15:1609-17.

11. Sun H, Liu C, Chen S, et al. Effect of surgical factors on the augmentation of cement-injectable cannulated pedicle screw fixation by a novel calcium phosphate-based nanocomposite. Front Med 2019;13:590-601.

12. Ulusoy OL, Kahraman S, Karalok I, et al. Pulmonary cement embolism following cement-augmented fenestrated pedicle screw fixation in adult spinal deformity patients with severe osteoporosis (analysis of 2978 fenestrated screws). Eur Spine J 2018;27:2348-56.

13. Uehara M, Takahashi J, Ikegami S, et al. Pedicle Screw Loosening After Posterior Spinal Fusion for Adolescent Idiopathic Scoliosis in Upper and Lower Instrumented Vertebrae Having Major Perforation. Spine (Phila Pa 1976) 2017;42:1895-900.

14. Li T, Shi L, Luo Y, et al. One-Level or Multilevel Interbody Fusion for Multilevel Lumbar Degenerative Diseases: A Prospective Randomized Control Study with a 4-Year Follow-Up. World Neurosurg 2018;110:e815-e822.

15. Guo HZ, Tang YC, Li YX, et al. The Effect and Safety of Polymethylmethacrylate-Augmented Sacral Pedicle Screws Applied in Osteoporotic Spine with Lumbosacral Degenerative Disease: A 2-Year Follow-up of 25 Patients. World Neurosurg 2019;121:e404-10.

16. Wang Md K, Jiang PhD C, Wang PhD L, et al. The biomechanical influence of anterior vertebral body osteophytes on the lumbar spine: A finite element study. Spine J 2018;18:2288-96.

17. Yan JZ, Wu ZH, Wang XS, et al. Finite element analysis on stress change of lumbar spine. Zhonghua Yi Xue Za Zhi 2009;89:1162-5.

18. Polikeit A, Nolte LP, Ferguson SJ. The effect of cement augmentation on the load transfer in an osteoporotic functional spinal unit: finite-element analysis. Spine (Phila Pa 1976) 2003;28:991-6.

19. $\mathrm{Xu} \mathrm{H}, \mathrm{Ju} \mathrm{W}, \mathrm{Xu} \mathrm{N}$, et al. Biomechanical comparison of transforaminal lumbar interbody fusion with 1 or 2 cages by finite-element analysis. Neurosurgery 2013;73:ons198205; discussion ons 205 .

20. Jones AC, Wilcox RK. Finite element analysis of the spine: towards a framework of verification, validation and sensitivity analysis. Med Eng Phys 2008;30:1287-304.

21. Yamamoto I, Panjabi MM, Crisco T, et al. Threedimensional movements of the whole lumbar spine and lumbosacral joint. Spine (Phila Pa 1976) 1989;14:1256-60. 
22. Shim CS, Park SW, Lee SH, et al. Biomechanical evaluation of an interspinous stabilizing device, Locker. Spine (Phila Pa 1976) 2008;33:E820-7.

23. Huang YP, Du CF, Cheng CK, et al. Preserving Posterior Complex Can Prevent Adjacent Segment Disease following Posterior Lumbar Interbody Fusion Surgeries: A Finite Element Analysis. PLoS One 2016;11:e0166452.

24. Yue JJ. Motion Preservation Surgery of the Spine: Advanced Techniques and Theory. Elsevier (Singapore) Pte Ltd., 2008:68.

25. Kobayashi K, Ando K, Nishida Y, et al. Epidemiological trends in spine surgery over 10 years in a multicenter database. Eur Spine J 2018;27:1698-703.

26. Thirukumaran CP, Raudenbush B, Li Y, et al. National Trends in the Surgical Management of Adult Lumbar Isthmic Spondylolisthesis: 1998 to 2011. Spine (Phila Pa 1976) 2016;41:490-501.

27. Cortesi PA, Assietti R, Cuzzocrea F, et al. Epidemiologic and Economic Burden Attributable to First Spinal Fusion Surgery: Analysis From an Italian Administrative Database. Spine (Phila Pa 1976) 2017;42:1398-404.

28. Fan HT, Zhang RJ, Shen CL, et al. The Biomechanical Properties of Pedicle Screw Fixation Combined With Trajectory Bone Cement Augmentation in Osteoporotic Vertebrae. Clin Spine Surg 2016;29:78-85.

Cite this article as: Guo HZ, Guo DQ, Tang YC, Liang D, Zhang SC. Selective cement augmentation of cranial and caudal pedicle screws provides comparable stability to augmentation on all segments in the osteoporotic spine: a finite element analysis. Ann Transl Med 2020;8(21):1384. doi: 10.21037/atm-202246
29. Girardo M, Cinnella P, Gargiulo G, et al. Surgical treatment of osteoporotic thoraco-lumbar compressive fractures: the use of pedicle screw with augmentation PMMA. Eur Spine J 2017;26:546-51.

30. Leichtle CI, Lorenz A, Rothstock S, et al. Pull-out strength of cemented solid versus fenestrated pedicle screws in osteoporotic vertebrae. Bone Joint Res 2016;5:419-26.

31. Erdem MN, Karaca S, Sar1 S, et al. Application of cement on strategic vertebrae in the treatment of the osteoporotic spine. Spine J 2017;17:328-37.

32. Elder BD, Lo SF, Holmes C, et al. The biomechanics of pedicle screw augmentation with cement. Spine J 2015;15:1432-45.

33. Mueller JU, Baldauf J, Marx S, et al. Cement leakage in pedicle screw augmentation: a prospective analysis of 98 patients and 474 augmented pedicle screws. J Neurosurg Spine 2016;25:103-9.

34. El Saman A, Kelm A, Meier S, et al. Intraoperative PEEPventilation during PMMA-injection for augmented pedicle screws: improvement of leakage rate in spinal surgery. Eur J Trauma Emerg Surg 2013;39:461-8.

35. Wu JC, Huang WC, Tsai HW, et al. Pedicle screw loosening in dynamic stabilization: incidence, risk, and outcome in 126 patients. Neurosurg Focus 2011;31:E9. 